\title{
Generalized Variational Principles on Oscillation for Nonlinear Nonhomogeneous Differential Equations
}

\author{
Jing Shao ${ }^{1}$ and Fanwei Meng ${ }^{2}$ \\ ${ }^{1}$ Department of Mathematics, Jining University, Qufu 273155, China \\ ${ }^{2}$ School of Mathematical Sciences, Qufu Normal University, Qufu 273165, China \\ Correspondence should be addressed to Jing Shao, shaojing99500@163.com \\ Received 30 June 2010; Revised 28 December 2010; Accepted 18 January 2011 \\ Academic Editor: Allan C. Peterson
}

Copyright (c) $2011 \mathrm{~J}$. Shao and F. Meng. This is an open access article distributed under the Creative Commons Attribution License, which permits unrestricted use, distribution, and reproduction in any medium, provided the original work is properly cited.

Using the generalized variational principle and the Riccati technique, new oscillation criteria are established for the forced second-order nonlinear differential equation, which improves and generalizes some of the new results in literature.

\section{Introduction}

In this paper, we consider the oscillatory behavior of the nonlinear nonhomogeneous differential equation of the form

$$
\left(p(t) \Psi(y(t))\left|y^{\prime}(t)\right|^{\alpha-1} y^{\prime}(t)\right)^{\prime}+q(t) f(y(t))=e(t), \quad t \geq t_{0}
$$

where $\alpha$ is a positive constant, $p, q, e \in C\left(\left[t_{0}, \infty\right), \mathbb{R}\right)$ with $p(t)>0, \Psi \in C(\mathbb{R},(0, \infty)), f \in$ $C(\mathbb{R}, \mathbb{R})$ satisfying $u f(u)>0$ for $u \neq 0$.

As usual, by a solution of (1.1) we mean a function $y \in C^{1}\left[T_{y}, \infty\right), T_{y} \geq t_{0}$, where $T_{y} \geq t_{0}$ depends on the particular solution, which has the property $p(t) \Psi(y(t))\left|y^{\prime}(t)\right|^{\alpha-1} y^{\prime}(t) \in$ $C^{1}\left[T_{y}, \infty\right)$ and satisfies (1.1). A nontrivial solution of (1.1) is called oscillatory if it has arbitrarily large zeros; otherwise, it is said to be nonoscillatory. Equation (1.1) is said to be oscillatory if all its solutions are oscillatory.

Recently, many research works have been done on the oscillatory and asymptotic behavior of solutions of the nonlinear nonhomogeneous differential equation of the form 
(1.1) or its special cases (see [1-7] and references cited therein). Using the variational method, oscillation criteria are obtained by Wong [1] for the forced linear differential equation, by Li and Cheng [2] for the forced half-linear differential equation, by Zheng and Meng [3] for the forced quasilinear differential equation, by Çakmak and Tiryaki [4] as well as Zheng and Cheng [5] for the forced nonlinear differential equation (some deficiencies in [2,4] are pointed out by Zheng and Meng [3]), and Erbe et al. [7], as well as Saker [8, 9] for the dynamic equation on time scales.

Meanwhile, in [6], Komkov gave a generalized Leighton's variational principle, which can also be used to obtain the oscillation criterion.

Theorem 1.1. Suppose that there exist a $C^{1}$ function $u(t)$ defined on $\left[s_{1}, t_{1}\right]$ and a function $G(u)$ such that $G(u(t))$ is not constant on $\left[s_{1}, t_{1}\right], G\left(u\left(s_{1}\right)\right)=G\left(u\left(t_{1}\right)\right)=0, g(u)=G^{\prime}(u)$ is continuous,

$$
\int_{s_{1}}^{t_{1}}\left[q(t) G(u(t))-p(t)\left(u^{\prime}(t)\right)^{2}\right] d t>0
$$

and $(g(u(t)))^{2} \leq 4 G(u(t))$ for $t \in\left[s_{1}, t_{1}\right]$. Then each solution of the equation

$$
\left(p(t) y^{\prime}(t)\right)^{\prime}+q(t) y(t)=0
$$

vanishes at least once on $\left[s_{1}, t_{1}\right]$.

The purpose of our paper is to use the generalized variational principle to study the oscillation for (1.1). These oscillation criteria are closely related to the generalized variational formulae (1.2), which improve the results mentioned above. Examples will also be given to illustrate the effectiveness of our main results.

Before going into the main results, let us state three sets of conditions commonly used in the literature which we rely on:

$$
\begin{aligned}
& \text { (S1) } 0<\Psi(u) \leq M, \quad\left|f^{\prime}(u)\right| \geq K|f(u)|^{(\beta-1) / \beta}>0, \quad \text { for } u \neq 0, \\
& \text { (S2) } \frac{\left|f^{\prime}(u)\right|}{\left[\Psi(u)|f(u)|^{\beta-1}\right]^{1 / \beta}} \geq \gamma>0, \quad \text { for } u \neq 0, \\
& \text { (S3) } 0<\Psi(u) \leq M, \quad \frac{|f(u)|}{|u|^{\beta}} \geq \delta>0, \quad \text { for } u \neq 0 .
\end{aligned}
$$

Here, $M, K>0,0<\alpha \leq \beta$, and $\gamma, \delta>0$ are constants. It is clear that assumption (S1) implies (S2), but not conversely. For example, the function $f(u)=u^{3}, \Psi(u)=u^{2}$ and $\beta=1$ do not satisfy (S1), but (S2) holds. In (S1) and (S2), we need $f$ to be differentiable. Clearly, this condition is not required in (S3). These differences force us to study (1.1) under the assumptions (S1), (S2), and (S3) in separate manners.

\section{The Case Where $\beta=\alpha$}

Firstly, we give an inequality, which is a transformation of Young's inequality. 
Lemma 2.1 (see [10]). Suppose that $X$ and $Y$ are nonnegative. Then

$$
\lambda X Y^{\lambda-1}-X^{\lambda} \leq(\lambda-1) Y^{\lambda}, \quad \lambda>1
$$

where the equality holds if and only if $X=Y$.

Now, we will give our main results.

Theorem 2.2. Assume (S2) holds. Suppose further that for any $T \geq t_{0}$, there exist $T \leq s_{1}<t_{1} \leq s_{2}<$ $t_{2}$ such that

$$
e(t) \begin{cases}\leq 0, & t \in\left[s_{1}, t_{1}\right] \\ \geq 0, & t \in\left[s_{2}, t_{2}\right] .\end{cases}
$$

Let $u \in C^{1}\left[s_{i}, t_{i}\right]$, and nonnegative functions $G_{1}, G_{2}$ satisfying $G_{i}\left(u\left(s_{i}\right)\right)=G_{i}\left(u\left(t_{i}\right)\right)=0, g_{i}(u)=$ $G_{i}^{\prime}(u)$ are continuous and $\left(g_{i}(u(t))\right)^{\alpha+1} \leq(\alpha+1)^{\alpha+1} G_{i}^{\alpha}(u(t))$ for $t \in\left[s_{i}, t_{i}\right], i=1,2$. If there exists $a$ positive function $\rho \in C^{1}\left(\left[t_{0}, \infty\right), \mathbb{R}\right)$ such that

$$
Q_{i}^{\rho}(u):=\int_{S_{i}}^{t_{i}} \rho(t)\left[q(t) G_{i}(u(t))-\left(\frac{\alpha}{\gamma}\right)^{\alpha} p(t)\left(\left|u^{\prime}(t)\right|+\frac{G_{i}^{1 /(\alpha+1)}(u(t))\left|\rho^{\prime}(t)\right|}{(\alpha+1) \rho(t)}\right)^{\alpha+1}\right] d t>0,
$$

for $i=1,2$. Then (1.1) is oscillatory.

Proof. Suppose to the contrary that there is a nonoscillatory solution $y(t)$ of (1.1). First, we consider the case when $y(t)>0$ eventually. Assume that $y(t)>0$ on $\left[T_{0}, \infty\right)$ for some $T_{0} \geq t_{0}$. Set

$$
w(t)=\frac{\rho(t) p(t) \Psi(y(t))\left|y^{\prime}(t)\right|^{\alpha-1} y^{\prime}(t)}{f(y(t))}, \quad t \geq T_{0}
$$

Then differentiating (2.4) and making use of (1.1), it follows that for all $t \geq T_{0}$,

$$
w^{\prime}(t)=-\rho(t) q(t)+\frac{\rho(t) e(t)}{f(y(t))}+\frac{\rho^{\prime}(t)}{\rho(t)} w(t)-\frac{|w(t)|^{(\alpha+1) / \alpha} f^{\prime}(y(t))}{\left[p(t) \rho(t) \Psi(y(t))|f(y(t))|^{\alpha-1}\right]^{1 / \alpha}}
$$

By assumptions, we can choose $s_{1}, t_{1} \geq T_{0}$ with $s_{1}<t_{1}$ so that $e(t) \leq 0$ on the interval $I_{1}=$ $\left[s_{1}, t_{1}\right]$. For $t \in I_{1}$ and in view of (1.5) and (2.5), $w(t)$ satisfies the inequality

$$
\rho(t) q(t) \leq-w^{\prime}(t)+\frac{\rho^{\prime}(t)}{\rho(t)} w(t)-\gamma \frac{|w(t)|^{(\alpha+1) / \alpha}}{p^{1 / \alpha}(t) \rho^{1 / \alpha}(t)} .
$$


Multiplying $G_{1}(u(t))$ through (2.6) and integrating (2.6) from $s_{1}$ to $t_{1}$, using the fact that $G_{1}\left(u\left(s_{1}\right)\right)=G_{1}\left(u\left(t_{1}\right)\right)=0$, we obtain

$$
\begin{aligned}
\int_{s_{1}}^{t_{1}} G_{1}(u(t)) \rho(t) q(t) d t \leq & \int_{s_{1}}^{t_{1}} G_{1}(u(t))\left[-w^{\prime}(t)+\frac{\rho^{\prime}(t)}{\rho(t)} w(t)-\frac{\gamma|w(t)|^{(\alpha+1) / \alpha}}{p^{1 / \alpha}(t) \rho^{1 / \alpha}(t)}\right] d t \\
= & -\left.G_{1}(u(t)) w(t)\right|_{s_{1}} ^{t_{1}}+\int_{s_{1}}^{t_{1}}\left[G_{i}(u(t))\right]^{\prime} w(t) d t \\
& +\int_{s_{1}}^{t_{1}} G_{1}(u(t)) \frac{\rho^{\prime}(t)}{\rho(t)} w(t) d t-\int_{s_{1}}^{t_{1}} G_{1}(u(t)) \frac{\gamma|w(t)|^{(\alpha+1) / \alpha}}{p^{1 / \alpha}(t) \rho^{1 / \alpha}(t)} d t \\
\leq & \int_{s_{1}}^{t_{1}}\left[\left|g_{1}(u(t))\right|\left|u^{\prime}(t)\right|+G_{1}(u(t)) \frac{\left|\rho^{\prime}(t)\right|}{\rho(t)}\right]|w(t)| d t \\
& -\gamma \int_{s_{1}}^{t_{1}} G_{1}(u(t)) \frac{|w(t)|^{(\alpha+1) / \alpha}}{p^{1 / \alpha}(t) \rho^{1 / \alpha}(t)} d t \\
\leq & (\alpha+1) \int_{s_{1}}^{t_{1}}\left[\left|G_{1}^{\alpha /(\alpha+1)}(u(t))\right|\left|u^{\prime}(t)\right|+G_{1}(u(t)) \frac{\left|\rho^{\prime}(t)\right|}{(\alpha+1) \rho(t)}\right]|w(t)| d t \\
& -\gamma \int_{s_{1}}^{t_{1}} G_{1}(u(t)) \frac{|w(t)|^{(\alpha+1) / \alpha}}{p^{1 / \alpha}(t) \rho^{1 / \alpha}(t)} d t .
\end{aligned}
$$

Let

$$
\begin{gathered}
X=\frac{\gamma^{\alpha /(\alpha+1)}}{p^{1 /(\alpha+1)}(t) \rho^{1 /(\alpha+1)}(t)} G_{1}^{\alpha /(\alpha+1)}(u(t))|w(t)|, \quad \lambda=1+\frac{1}{\alpha} \\
Y=\frac{\alpha^{\alpha} p^{\alpha /(\alpha+1)}(t) \rho^{\alpha /(\alpha+1)}(t)}{\gamma^{\alpha^{2} /(\alpha+1)}}\left[\left|u^{\prime}(t)\right|+\frac{G_{1}^{1 /(\alpha+1)}(u(t))\left|\rho^{\prime}(t)\right|}{(\alpha+1) \rho(t)}\right]^{\alpha} .
\end{gathered}
$$

By Lemma 2.1 and (2.7), we have

$$
\int_{s_{1}}^{t_{1}} G_{1}(u(t)) \rho(t) q(t) d t \leq \int_{s_{1}}^{t_{1}}\left(\frac{\alpha}{\gamma}\right)^{\alpha} p(t) \rho(t)\left(\left|u^{\prime}(t)\right|+\frac{G_{1}^{1 /(\alpha+1)}(u(t))\left|\rho^{\prime}(t)\right|}{(\alpha+1) \rho(t)}\right)^{\alpha+1} d t
$$

which contradicts (2.3) with $i=1$.

When $y(t)<0$ holds eventually, we assume $y(t)<0$ for $t \geq T_{0}>t_{0}$. Defining the Riccati transformation as (2.4), we get that (2.5) is true. In this case, we choose $t_{2}>s_{2} \geq T_{0}$ so that $e(t) \geq 0$ on the interval $I_{2}=\left[s_{2}, t_{2}\right]$. For a given $t \in I_{2},(1.5)$ and (2.5) imply (2.6). Multiplying $G_{2}(u(t))$ through (2.6) and integrating (2.6) from $s_{2}$ to $t_{2}$, using the fact that $G_{2}\left(u\left(s_{2}\right)\right)=G_{2}\left(u\left(t_{2}\right)\right)=0$, we obtain a similar contradiction to (2.3) with $i=2$. This completes the proof. 
Corollary 2.3. If $\rho(t) \equiv 1$ in Theorem 2.2 and (2.3) is replaced by

$$
Q_{i}(u):=\int_{s_{i}}^{t_{i}}\left[q(t) G_{i}(u(t))-\left(\frac{\alpha}{\gamma}\right)^{\alpha} p(t)\left|u^{\prime}(t)\right|^{\alpha+1}\right] d t>0
$$

for $i=1,2$, then (1.1) is oscillatory.

Remark 2.4. The left side of inequality (2.10) is closely related to the generalized variational formulae (1.2). Particularly, we obtain $\alpha=\gamma$ for the linear equation; thus, (2.10) reduces to (1.2) for the linear differential equation. So our Theorem 2.2 and Corollary 2.3 are generalizations of the paper by Zheng and Cheng [5] and improvement of papers by Li and Cheng [2] and by Çakmak and Tiryaki [4].

Theorem 2.5. Assume that (S3) holds. Suppose further that for any $T \geq t_{0}$, there exist $T \leq s_{1}<$ $t_{1} \leq s_{2}<t_{2}$ such that (2.2) holds. Let $u \in C^{1}\left[s_{i}, t_{i}\right]$, and nonnegative functions $G_{1}, G_{2}$ satisfying $G_{i}\left(u\left(s_{i}\right)\right)=G_{i}\left(u\left(t_{i}\right)\right)=0, g_{i}(u)=G_{i}^{\prime}(u)$ are continuous and $\left(g_{i}(u(t))\right)^{\alpha+1} \leq(\alpha+1)^{\alpha+1} G_{i}^{\alpha}(u(t))$ for $t \in\left[s_{i}, t_{i}\right], i=1$, If If there exists a positive function $\rho \in C^{1}\left(\left[t_{0}, \infty\right), \mathbb{R}\right)$ such that

$$
Q_{i}^{\rho}(u):=\int_{s_{i}}^{t_{i}} \rho(t)\left[\delta q(t) G_{i}(u(t))-M p(t)\left(\left|u^{\prime}(t)\right|+\frac{G_{i}^{1 /(\alpha+1)}(u(t))\left|\rho^{\prime}(t)\right|}{(\alpha+1) \rho(t)}\right)^{\alpha+1}\right] d t>0,
$$

for $i=1,2$. Then (1.1) is oscillatory.

Proof. Suppose to the contrary that there is a nonoscillatory solution $y(t)$. Firstly, we assume that $y(t)>0$ on $\left[T_{0}, \infty\right)$ for some $T_{0} \geq t_{0}$. Set

$$
w(t)=\frac{\rho(t) p(t) \Psi(y(t))\left|y^{\prime}(t)\right|^{\alpha-1} y^{\prime}(t)}{|y(t)|^{\alpha-1} y(t)}, \quad t \geq T_{0}
$$

Then differentiating (2.12) and making use of (1.1) and (S3), we see that for all $t \geq T_{0}$, we have

$$
\begin{aligned}
w^{\prime}(t) & =\frac{-q(t) \rho(t) f(y(t))}{|y(t)|^{\alpha-1} y(t)}+\frac{\rho(t) e(t)}{|y(t)|^{\alpha-1} y(t)}+\frac{\rho^{\prime}(t) w(t)}{\rho(t)}-\alpha \frac{|w(t)|^{(\alpha+1) / \alpha}}{[\rho(t) p(t) \Psi(y(t))]^{1 / \alpha}} \\
& \leq-\delta q(t) \rho(t)+\frac{\rho^{\prime}(t) w(t)}{\rho(t)}+\frac{e(t) \rho(t)}{|y(t)|^{\alpha-1} y(t)}-\frac{\alpha}{M^{1 / \alpha}} \frac{|w(t)|^{(\alpha+1) / \alpha}}{\rho^{1 / \alpha}(t) p^{1 / \alpha}(t)} .
\end{aligned}
$$


By assumptions, we choose $s_{1}, t_{1} \geq T_{0}$ so that $e(t) \leq 0$ on the interval $I_{1}=\left[s_{1}, t_{1}\right]$. For $t \in I_{1}$, (2.13) implies that $w(t)$ satisfies the inequality

$$
\delta \rho(t) q(t) \leq-w^{\prime}(t)+\frac{\rho^{\prime}(t) w(t)}{\rho(t)}-\frac{\alpha}{M^{1 / \alpha}} \frac{|w(t)|^{(\alpha+1) / \alpha}}{p^{1 / \alpha}(t) \rho(t)^{1 / \alpha}(t)} .
$$

A similar method as to that of Theorem 2.2 implies a contradiction to (2.11) with $i=1$.

When $y(t)<0$ holds eventually, we assume $y(t)<0$ for $t \geq T_{0}>t_{0}$. Defining the Riccati transformation as (2.12), we get that (2.13) is true. In this case, we choose $t_{2}>s_{2} \geq T_{0}$ so that $e(t) \geq 0$ on the interval $I_{2}=\left[s_{2}, t_{2}\right]$. For a given $t \in I_{2}$, we get that (2.14) holds. A similar method reaches a similar contradiction to (2.11) with $i=2$. This completes the proof.

Now we give two examples to illustrate the efficiency of our results.

Example 2.6. Consider the following forced half-linear differential equation:

$$
\left(t^{\lambda}\left|y^{\prime}(t)\right|^{\alpha-1} y^{\prime}(t)\right)^{\prime}+K t^{\lambda}|y(t)|^{\alpha-1} y(t)=-\sin t
$$

for $t \geq 1$, where $K, \lambda>0$ are constants and $\alpha=1$. We may show that (2.15) is oscillatory for $K>2 e(1+\lambda / 2)^{2}$ using Theorem 2.2. Indeed, since the zeros of the forcing term $-\sin t$ are $n \pi$, the constant $\gamma$ in (1.5) is $\alpha$, that is, $\gamma=\alpha$. For any $T \geq 1$, we choose $n$ sufficiently large so that $n \pi=2 k \pi \geq T$ and $s_{1}=2 k \pi$ and $t_{1}=(2 k+1) \pi$. Selecting $u(t)=\sin t \geq 0, G_{1}(u)=u^{2} \exp (-u)$ (we note that $\left(G_{1}^{\prime}(u)\right)^{2} \leq 4 G_{1}(u)$ for $\left.u \geq 0\right), \rho(t)=t^{-\lambda}$, then we have

$$
\begin{aligned}
\int_{s_{1}}^{t_{1}} \rho(t) q(t) G_{1}(u(t)) d t=\int_{2 k \pi}^{(2 k+1) \pi} t^{-\lambda} K t^{\lambda}(\sin t)^{2} \exp (-\sin t) d t \\
\quad=K \int_{0}^{\pi}(\sin t)^{2} \exp (-\sin t) d t \\
\quad \geq \frac{K}{e} \int_{0}^{\pi} \frac{1-\cos 2 t}{2} d t=\frac{K \pi}{2 e} \\
\int_{S_{1}}^{t_{1}} \rho(t)\left(\frac{\alpha}{\gamma}\right)^{\alpha} p(t)\left(\left|u^{\prime}(t)\right|+\frac{G_{i}^{1 /(\alpha+1)}(u(t))\left|\rho^{\prime}(t)\right|}{(\alpha+1) \rho(t)}\right)^{\alpha+1} d t \\
\quad=\int_{2 k \pi}^{(2 k+1) \pi} t^{-\lambda}\left(\frac{\alpha}{\alpha}\right)^{\alpha} t^{\lambda}\left(|\cos t|+\frac{\lambda \sin t \exp (-\sin t / 2)}{t}\right)^{2} d t \\
\quad<\int_{0}^{\pi}\left(1+\frac{\lambda}{2}\right)^{2} d t=\left(1+\frac{\lambda}{2}\right)^{2} \pi .
\end{aligned}
$$

So we have $Q_{1}^{\rho}(u)>0$ provided $K>2 e(1+\lambda / 2)^{2}$. Similarly, for $s_{2}=(2 k+1) \pi$ and $t_{2}=$ $(2 k+2) \pi$, we select $u(t)=\sin t \leq 0, G_{2}(u)=u^{2} \exp (u)$, and we note that $\left(G_{2}^{\prime}(u)\right)^{2} \leq 4 G_{2}(u)$ for $u \leq 0$; we can show that the integral inequality $Q_{2}^{\rho}(u)>0$ for $K>2 e(1+\lambda / 2)^{2}$. So $(2.15)$ is oscillatory for $K>2 e(1+\lambda / 2)^{2}$ by Theorem 2.2 . 
Example 2.7. Consider the following forced half-linear differential equation:

$$
\left[(2+\cos t) t^{-\lambda}\left|y^{\prime}(t)\right|^{\alpha-1} y^{\prime}(t)\right]^{\prime}+K t^{-\lambda} \exp (\sin t)|y(t)|^{\alpha-1} y(t)=-\sin t,
$$

for $t \geq 1$, where $K, \lambda>0$ are constants and $\alpha=1$. We may show that (2.17) is oscillatory for $K>3(1+\lambda)^{2}$ using Theorem 2.2. Indeed, since the zeros of the forcing term $-\sin t$ are $n \pi$, the constant $\gamma$ in (1.5) is $\alpha$, that is, $\gamma=\alpha$. In fact, for any $T \geq 1$, we choose $n$ sufficiently large so that $n \pi=2 k \pi \geq T$ and $s_{1}=2 k \pi$ and $t_{1}=(2 k+1) \pi$. Selecting $u(t)=\sin t \geq 0$, $G_{1}(u)=u^{2} \exp (-u)$ (we note that $\left(G_{1}^{\prime}(u)\right)^{2} \leq 4 G_{1}(u)$ for $u \geq 0$ ), $\rho(t)=t^{\lambda}$, then we have

$$
\begin{aligned}
& \int_{s_{1}}^{t_{1}} \rho(t) q(t) G_{1}(u(t)) d t=\int_{2 k \pi}^{(2 k+1) \pi} t^{\lambda} K t^{-\lambda}(\sin t)^{2} \exp (-\sin t) d t \\
& \quad=K \int_{0}^{\pi}(\sin t)^{2} \exp (\sin t-\sin t) d t \\
& \quad \geq K \int_{0}^{\pi} \frac{1-\cos 2 t}{2} d t=\frac{K \pi}{2}, \\
& \int_{s_{1}}^{t_{1}} \rho(t)\left(\frac{\alpha}{\gamma}\right)^{\alpha} p(t)\left(\left|u^{\prime}(t)\right|+\frac{G_{i}^{1 /(\alpha+1)}(u(t))\left|\rho^{\prime}(t)\right|}{(\alpha+1) \rho(t)}\right)^{\alpha+1} d t \\
& \quad=\int_{2 k \pi}^{(2 k+1) \pi} t^{\lambda} \frac{1}{2}\left(\frac{\alpha}{\alpha}\right)^{\alpha}(2+\cos t) t^{-\lambda}\left(|\cos t|+\frac{\lambda \sin t \exp (-\sin t / 2)}{t}\right)^{2} d t \\
& \quad<\int_{0}^{\pi} \frac{3}{2}(1+\lambda)^{2} d t=\frac{3}{2}(1+\lambda)^{2} \pi .
\end{aligned}
$$

So, we have $Q_{1}^{\rho}(u)>0$ provided $K>3(1+\lambda)^{2}$. Similarly, for $s_{2}=(2 k+1) \pi$ and $t_{2}=(2 k+2) \pi$, we select $u(t)=\sin t \leq 0, G_{2}(u)=u^{2} \exp (u)$, and we note that $\left(G_{2}^{\prime}(u)\right)^{2} \leq 4 G_{2}(u)$ for $u \leq 0$; we can show that the integral inequality $Q_{2}^{\rho}(u)>0$ for $K>3(1+\lambda)^{2}$. So, (2.17) is oscillatory for $K>3(1+\lambda)^{2}$ by Theorem 2.2 .

\section{The Case Where $\beta>\alpha$}

We now handle the case where $\beta>\alpha$.

Theorem 3.1. Assume that (S3) holds. Suppose further that for any $T \geq t_{0}$, there exist $T \leq s_{1}<$ $t_{1} \leq s_{2}<t_{2}$ such that (2.2) holds. Let $u \in C_{1}\left[s_{i}, t_{i}\right]$, and the nonnegative functions $G_{1}, G_{2}$ satisfying $G_{i}\left(u\left(s_{i}\right)\right)=G_{i}\left(u\left(t_{i}\right)\right)=0, g_{i}(u)=G_{i}^{\prime}(u)$ are continuous and $\left(g_{i}(u(t))\right)^{\alpha+1} \leq(\alpha+1)^{\alpha+1} G_{i}^{\alpha}(u(t))$ for $t \in\left[s_{i}, t_{i}\right], i=1$, 2. If there exists a positive function $\rho \in C^{1}\left(\left[t_{0}, \infty\right), \mathbb{R}\right)$ such that

$$
Q_{i}^{\rho}(u):=\int_{S_{i}}^{t_{i}} \rho(t)\left[Q_{e}(t) G_{i}(u(t))-M p(t)\left(\left|u^{\prime}(t)\right|+\frac{G_{i}^{1 /(\alpha+1)}(u(t))\left|\rho^{\prime}(t)\right|}{(\alpha+1) \rho(t)}\right)^{\alpha+1}\right] d t>0,
$$


for $i=1,2$, then (1.1) is oscillatory, where

$$
Q_{e}(t)=\alpha^{-\alpha / \beta} \beta(\beta-\alpha)^{(\alpha-\beta) / \beta}[\delta q(t)]^{\alpha / \beta}|e(t)|^{(\beta-\alpha) / \beta} .
$$

Proof. Suppose to the contrary that there is a nontrivial nonoscillatory solution. Firstly, we assume that $y(t)>0$ on $\left[T_{0}, \infty\right)$ for some $T_{0} \geq t_{0}$. Set

$$
w(t)=\frac{\rho(t) p(t) \Psi(y(t))\left|y^{\prime}(t)\right|^{\alpha-1} y^{\prime}(t)}{|y(t)|^{\alpha-1} y(t)}, \quad t \geq T_{0} .
$$

Then differentiating (3.3) and making use of (1.1), it follows that for all $t \geq T_{0}$,

$$
\begin{aligned}
w^{\prime}(t) & =\frac{-q(t) \rho(t) f(y(t))}{|y(t)|^{\alpha-1} y(t)}+\frac{\rho(t) e(t)}{|y(t)|^{\alpha-1} y(t)}+\frac{\rho^{\prime}(t) w(t)}{\rho(t)}-\alpha \frac{|w(t)|^{(\alpha+1) / \alpha}}{[\rho(t) p(t) \Psi(y(t))]^{1 / \alpha}} \\
& =-\frac{q(t) \rho(t) f(y(t))}{|y(t)|^{\beta-1} y(t)}|y(t)|^{\beta-\alpha}+\frac{e(t) \rho(t)}{|y(t)|^{\alpha-1} y(t)}+\frac{\rho^{\prime}(t) w(t)}{\rho(t)}-\alpha \frac{|w(t)|^{(\alpha+1) / \alpha}}{[p(t) \rho(t) \Psi(y(t))]^{1 / \alpha}} \\
& \leq-\delta q(t) \rho(t)|y(t)|^{\beta-\alpha}+\frac{e(t) \rho(t)}{|y(t)|^{\alpha-1} y(t)}+\frac{\rho^{\prime}(t) w(t)}{\rho(t)}-\frac{\alpha}{M^{1 / \alpha}} \frac{|w(t)|^{(\alpha+1) / \alpha}}{p^{1 / \alpha}(t) \rho^{1 / \alpha}(t)} .
\end{aligned}
$$

By assumptions, we can choose $t_{1}>s_{1} \geq T_{0}$ so that $e(t) \leq 0$ on the interval $I_{1}=\left[s_{1}, t_{1}\right]$. For a given $t \in I_{1}$, set $F(x)=\delta q(t) x^{\beta-\alpha}-e(t) / x^{\alpha}$, and we have $F^{\prime}\left(x^{*}\right)=0, F^{\prime \prime}\left(x^{*}\right)>0$, where $x^{*}=[-\alpha e(t) /(\beta-\alpha) \delta q(t)]^{1 / \beta}$. So, $F(x)$ attains its minimum at $x^{*}$ and

$$
F(x) \geq F\left(x^{*}\right)=Q_{e}(t) .
$$

So (3.4) and (3.5) imply that $w(t)$ satisfies

$$
\rho(t) Q_{e}(t) \leq-w^{\prime}(t)-\frac{\alpha}{M^{1 / \alpha}} \frac{|w(t)|^{(\alpha+1) / \alpha}}{p^{1 / \alpha}(t) \rho^{1 / \alpha}(t)}+\frac{\rho^{\prime}(t) w(t)}{\rho(t)} .
$$

The remaining argument is the same as in the proof of Theorem 2.2, so we obtain a desired contradiction to (3.1) with $i=1$ when $y(t)>0$ eventually.

On the other hand, if $y(t)$ is a negative solution for $t \geq T_{0} \geq t_{0}$, we define the Riccati transformation (3.3) to yield (3.4). In this case, we choose $t_{2}>s_{2} \geq T_{0}$ so that $e(t) \geq 0$ on the interval $I_{2}=\left[s_{2}, t_{2}\right]$. For a given $t \in I_{2}$, set $F(x)=\delta q(t) x^{\beta-\alpha}-e(t) / x^{\alpha}$, and we have $F(x) \geq$ $F\left(x^{*}\right)=Q_{e}(t)$. The remaining proof is similar to that of Theorem 2.2; a desired contradiction to (3.1) with $i=2$ can be obtained. This completes the proof. 
Corollary 3.2. If $\rho(t) \equiv 1$ in Theorem 3.1 and the hypothesis (3.1) is replaced by

$$
\widetilde{Q}_{i}(u):=\int_{S_{i}}^{t_{i}}\left[Q_{e}(t) G_{i}(u(t))-M p(t)\left|u^{\prime}(t)\right|^{\alpha+1}\right] d t>0
$$

for $i=1,2$, then (1.1) is oscillatory.

We remark that Corollary 3.2 is closely related to the generalized variational formulae. Furthermore, in Theorem 3.1, there is no restriction on the positive constant $\alpha$, so Theorem 2.5 can be treated as its limiting case when $\beta \rightarrow \alpha+0$ with the convention that $0^{0}=1$.

Example 3.3. Consider the following forced quasilinear differential equation:

$$
\left(\gamma t^{\lambda / 3} y^{\prime}(t)\right)^{\prime}+t^{\lambda}|y(t)|^{2} y(t)=-\sin ^{3} t, \quad t \geq 1,
$$

where $\gamma, \lambda>0$ are constants. We see that $\Psi(u) \equiv 1$, which implies that $M=1$, and $\alpha=1$, $\beta=3$ in Theorem 3.1. Since $\alpha<\beta$, Theorem 2.2 cannot be applied. However, we can obtain oscillation for (3.8) using Theorem 3.1. For any $T \geq 1$, choose $n$ sufficiently large so that $n \pi=2 k \pi \geq T$ and $s_{1}=2 k \pi$ and $t_{1}=(2 k+1) \pi$. We choose $u(t)=\sin t \geq 0, G_{1}(u)=u^{2} \exp (-u)$ (we note that $\left(G_{1}^{\prime}(u)\right)^{2} \leq 4 G_{1}(u)$, for $u \geq 0$ ), $\rho(t)=t^{\lambda}$. In fact, we can easily verify that $Q_{e}(t)=(3 / 2) \sqrt[3]{2} t^{\lambda / 3} \sin ^{2} t$

$$
\begin{aligned}
\int_{s_{1}}^{t_{1}} Q_{e}(t) q(t) G_{1}(u(t)) d t=\int_{2 k \pi}^{(2 k+1) \pi} t^{-\lambda / 3} \frac{3}{2} \sqrt[3]{2} \sin t^{4} \exp (-\sin t) d t \\
\quad=\frac{3}{2} \sqrt[3]{2} \int_{0}^{\pi}(\sin t)^{4} \exp (-\sin t) d t \\
\quad \geq \frac{9 \sqrt[3]{2}}{16 e} \pi \\
\int_{s_{1}}^{t_{1}} \rho(t)\left(\frac{\alpha}{\gamma}\right)^{\alpha} M p(t)\left(\left|u^{\prime}(t)\right|+\frac{G_{i}^{1 /(\alpha+1)}(u(t))\left|\rho^{\prime}(t)\right|}{(\alpha+1) \rho(t)}\right)^{\alpha+1} d t \\
\quad=\int_{s_{1}}^{t_{1}} t^{-\lambda / 3} \gamma t^{\lambda / 3}\left(|\cos t|+\frac{(\lambda / 3) \sin t \exp (-\sin t / 2)}{2 t}\right)^{2} d t \\
\quad<\gamma\left(1+\frac{\lambda}{6}\right)^{2} \pi .
\end{aligned}
$$

So, we have that (3.1) is true for $i=1$ provided $0<\gamma<9 \sqrt[3]{2} / 16 e(1+\lambda / 6)^{2}$. Similarly, for $s_{2}=(2 k+1) \pi$ and $t_{2}=(2 k+2) \pi$, we can show that (3.1) is true for $i=2$. So (3.8) is oscillatory for $0<\gamma<9 \sqrt[3]{2} / 16 e(1+\lambda / 6)^{2}$ by Theorem 3.1. 


\section{Acknowledgments}

The authors sincerely thank the referees for their constructive suggestions and corrections. This research was partially supported by the NSF of China (Grant 10771118, 10801089), NSF of Shandong (Grant ZR2009AM011, ZR2009AQ010), and NSF of Jining University (Grant 2010QNKJ09).

\section{References}

[1] J. S. W. Wong, “Oscillation criteria for a forced second-order linear differential equation," Journal of Mathematical Analysis and Applications, vol. 231, no. 1, pp. 235-240, 1999.

[2] W.-T. Li and S. S. Cheng, "An oscillation criterion for nonhomogeneous half-linear differential equations," Applied Mathematics Letters, vol. 15, no. 3, pp. 259-263, 2002.

[3] Z. Zheng and F. Meng, "Oscillation criteria for forced second-order quasi-linear differential equations," Mathematical and Computer Modelling, vol. 45, no. 1-2, pp. 215-220, 2007.

[4] D. Çakmak and A. Tiryaki, "Oscillation criteria for certain forced second-order nonlinear differential equations," Applied Mathematics Letters, vol. 17, no. 3, pp. 275-279, 2004.

[5] Z. Zheng and S. S. Cheng, "Variational oscillation criteria for nonlinear nonhomogeneous differential equations," Applied Mathematics E-Notes, vol. 7, pp. 247-256, 2007.

[6] V. Komkov, "A generalization of Leighton's variational theorem," Applicable Analysis, vol. 1, pp. 377$383,1972$.

[7] L. Erbe, A. Peterson, and S. H. Saker, "Oscillation criteria for a forced second-order nonlinear dynamic equation," Journal of Difference Equations and Applications, vol. 14, no. 10-11, pp. 997-1009, 2008.

[8] S. H. Saker, "Boundedness of solutions of second-order forced nonlinear dynamic equations," The Rocky Mountain Journal of Mathematics, vol. 36, no. 6, pp. 2027-2039, 2006.

[9] S. H. Saker, "Oscillation of second-order forced nonlinear dynamic equations on time scales," Electronic Journal of Qualitative Theory of Differential Equations, vol. 3, pp. 1-17, 2005.

[10] G. H. Hardy, J. E. Littlewood, and G. Pólya, Inequalities, Cambridge Mathematical Library, Cambridge University Press, Cambridge, Mass, USA, 2nd edition, 1988. 


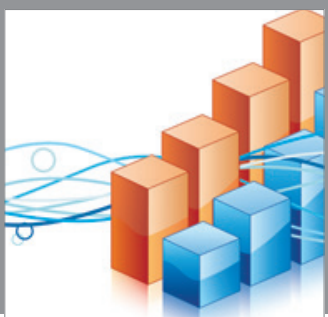

Advances in

Operations Research

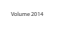

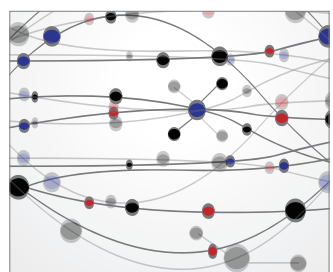

\section{The Scientific} World Journal
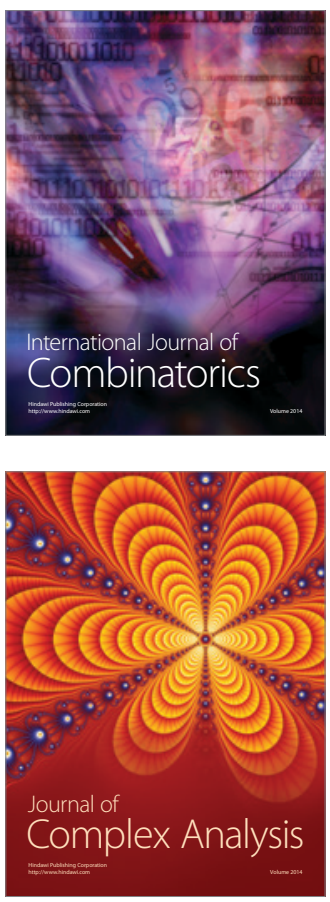

International Journal of

Mathematics and

Mathematical

Sciences
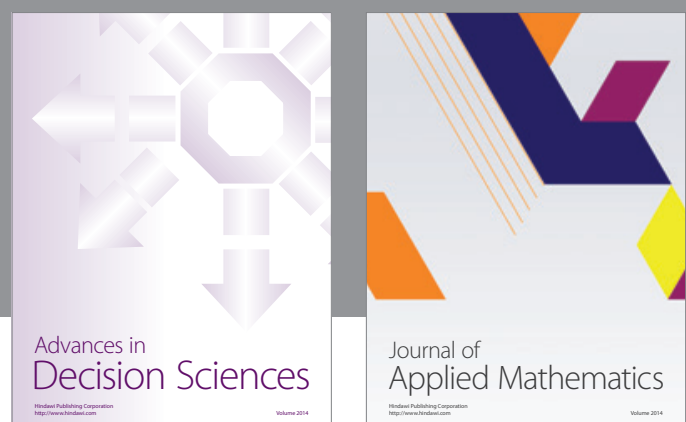

Journal of

Applied Mathematics
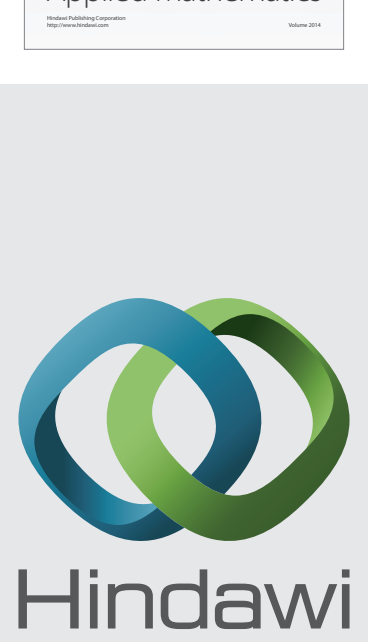

Submit your manuscripts at http://www.hindawi.com
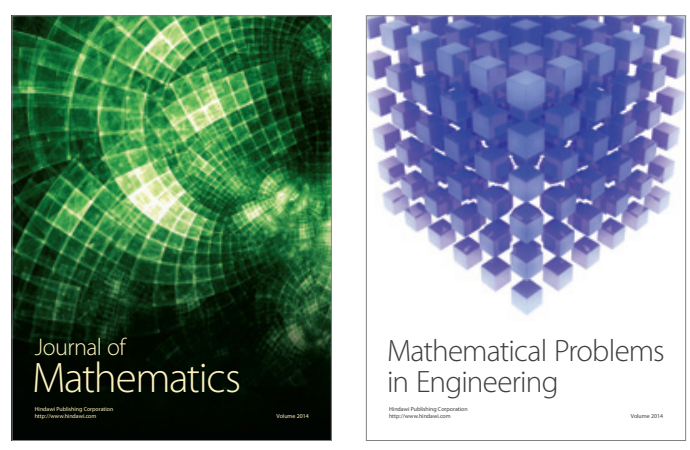

Mathematical Problems in Engineering
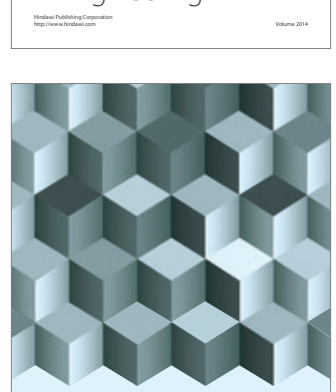

Journal of

Function Spaces
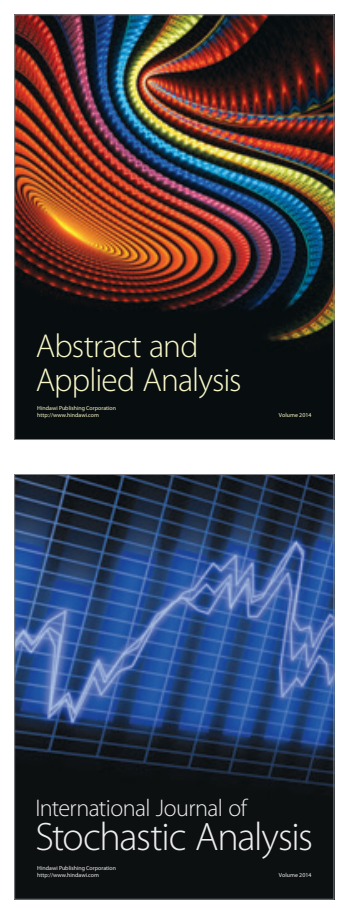

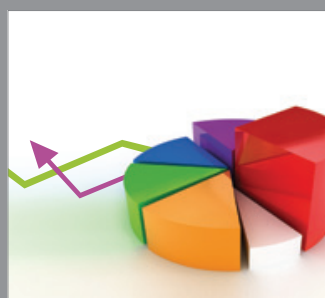

ournal of

Probability and Statistics

Promensencen
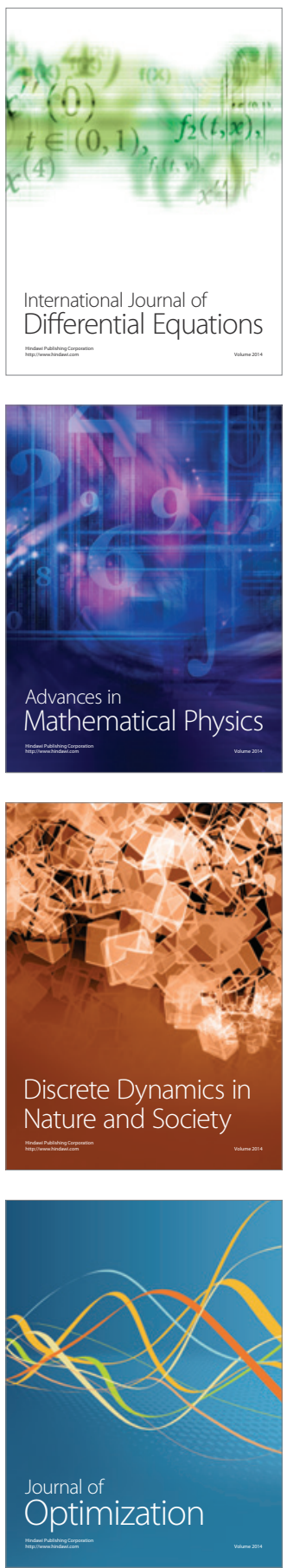